\title{
Role of microRNA508-3p in melanogenesis by targeting microphthalmia transcription factor in melanocytes of alpaca
}

\author{
J. Zhang ${ }^{1}$, Y. Liu ${ }^{1}$, Z. Zhu ${ }^{2}$, S. Yang ${ }^{1}$, K. Ji ${ }^{1}$, S. Hu ${ }^{1}$, X. Liu ${ }^{1}$, J. Yao ${ }^{3}$, R. Fan ${ }^{1 \dagger}$ and C. Dong ${ }^{1}$ \\ ${ }^{1}$ College of Animal Science and Veterinary Medicine, Shanxi Agricultural University, Taigu 030801, China; ${ }^{2}$ College of Life Science, Shanxi Agricultural University, \\ Taigu 030801, China; ${ }^{3}$ Division of Animal and Nutritional Sciences, West Virginia University, Morgantown, WV 26506, USA
}

(Received 20 January 2016; Accepted 31 May 2016; First published online 13 July 2016)

It has been demonstrated that microRNAs (miRNAs) play important roles in the control of melanogenesis and hair color in mammals. By comparing miRNA expression profiles between brown and white alpaca skin, we previously identified miR508-3p as a differentially expressed miRNA suggesting its potential role in melanogenesis and hair color formation. The present study was conducted to determine the role of miR508-3p in melanogenesis in alpaca melanocytes. In situ hybridization showed that miR508-3p is abundantly present in the cytoplasma of alpaca melanocytes. miR508-3p was predicted to target the gene encoding microphthalmia transcription factor (MITF) and a luciferase reporter assay indicated that miR508-3p regulates MITF expression by directly targeting its 3'UTR. Overexpression of miR508-3p in alpaca melanocytes down-regulated MITF expression both at the messenger RNA and protein level and resulted in decreased expression of key melanogenic genes including tyrosinase and tyrosinase-related protein 2. Overexpression of miR508-3p in melanocytes also resulted in decreased melanin production including total alkali-soluble melanogenesis, eumelanogenesis and pheomelanogenesis. Results support a functional role of miR508-3p in regulating melanogenesis in alpaca melanocytes by directly targeting MITF.

Keywords: miR508-3p, MITF, melanocytes, melanin, alpaca

\section{Implication}

Alpaca is an important fiber-producing species with 22 different natural hair colors. Understanding the molecular mechanism of hair color formation in this species is of interest to the alpaca breeders. This study revealed a functional role of miR508-3p in melanogenesis in alpaca melanocytes providing a new candidate gene for producing natural hair colors in fine fiber-producing species such as alpaca by transgenic approach.

\section{Introduction}

Many coding genes have been reported to play important roles in hair color formation in mammalian species. microRNAs (miRNAs) are non-coding 21 to $25 \mathrm{nt}$ RNA molecules, that play important roles in regulating gene expression in development, physiology and disease of animals (Bartel, 2004). The expression and function of miRNAs in the skin of mammalian species including mouse (Andl et al., 2006; Yi et al., 2006; Dong et al., 2012), goat and sheep (Wenguang et al., 2007), and alpaca (Zhu et al., 2010;

${ }^{\dagger}$ E-mail: ruiwenfan@163.com
Yang et al., 2015) have been reported. For example, overexpression of miR-137 in mice causes a change in skin color from black to brown (Dong et al., 2012). Other miRNAs, such as miR-340, miR182 and miR-25, were shown to regulate pigmentation by targeting microphthalmia transcription factor (MITF) messenger RNA (mRNA) (Bemis et al., 2008; Segura et al., 2009; Goswami et al., 2010). miR508-3p is one of the members of the miR-506 family, which are located on Xq27.3 (Zhou et al., 2010). miR508-3p plays an important role as a tumor suppressor during tumor formation (Zhai et al., 2012). Overexpression of miR508-3p and miR-509-3p suppresses the proliferation of renal cell carcinoma, induces cell apoptosis, and inhibits cell migration in vitro (Zhai et al., 2012). From the miRNA profiles of alpaca skins with white hair color $v$. brown hair color, we found miR508-3p was expressed differentially (Tian et al., 2012), which suggested that miR508-3p might be related to hair color. Moreover, bioinformatics analysis revealed that one of the predicted target genes of miR508-3p is MITF. MITF is a basic helixloop-helix leucine zipper transcription factor that regulates melanocyte cellular differentiation and the transcription of melanogenic enzymes (tyrosinase (TYR), tyrosinase-related protein TYRP1 and TYRP2) (Levy et al., 2006). MITF not only 
controls expression of pigmentation-related genes but also conveys hormonal and stress responses to pigmentation genes (Vachtenheim and Borovanský, 2010). Therefore, we hypothesized that miR508-3p may regulate melanin production by targeting MITF. This study was undertaken to determine the role of miR508-3p in melanogenesis using cultured alpaca melanocytes.

\section{Material and methods}

\section{In situ hybridization}

Alpaca melanocytes used in this study were established by our lab and were maintained as previously described (Bai et al., 2010). For in situ hybridization, cells were fixed in $4 \%$ paraformaldehyde for $30 \mathrm{~min}$, followed by washing in $0.1 \mathrm{M}$ PBS (Phosphate Buffer Saline) (pH 7.4) three times. After treatment with proteinase $\mathrm{K}(40 \mathrm{~g} / \mathrm{ml}$; Roche Applied Science, Indianapolis, IN, USA), cells were fixed in $1 \%$ paraformaldehyde for $10 \mathrm{~min}$. A quantity of 3 pmol of digoxigenin-labeled miR508-3p probe were diluted into $20 \mu \mathrm{l}$ of hybridization buffer, applied to the slides, and allowed to hybridize at $37^{\circ} \mathrm{C}$ overnight. Slides were then washed at $37^{\circ} \mathrm{C}$ with $2 \times$ SSC (Saline Sodium Citrate) solution and incubated with 1:1000 diluted alkaline phosphatase-conjugated mouse anti-digoxigenin antibody (Roche Applied Science) for $2 \mathrm{~h}$ at $37^{\circ} \mathrm{C}$. Alkaline phosphatase reaction was carried out with diaminobenzidine (DAB). The miR508-3p probe sequence is $5^{\prime}$-ATTGTCACTTTTTGGAGTA GA-3', and scrambled sequence is 5'-GTGTAACACGTCTATA CGCCCA-3'(Bio-High Technology, Shijiazhuang, Hebei, China).

\section{Construction of plasmids}

The miR508-3p expression plasmid was constructed by inserting an oligonucleotide corresponding to the sequence of the pre-miR508-3p into a mammalian expression vector, pcDNA6.2-GW/EmGFPmiR (Invitrogen, Shanghai, China), which contains a CMV (Cytomegalovirus) promoter driving the expression of GFP (Green Fluorescent Protein) and miR508-3p. A negative control (NC) plasmid was also constructed using scrambled sequence of pre-miR508-3p. The luciferase reporter plasmid was constructed by cloning $3^{\prime}$ UTR (untranslated regions) of alpaca MITF into a dual luciferase pmirGLO vector (Promega, Madison, USA). 3'UTR sequence of alpaca MITF containing the miR508-3p binding site was obtained by PCR using alpaca skin complementary DNA (CDNA) as a template with primers containing $X b a l$ and Notl sites (Table 1). The PCR product and the vector were digested with $\mathrm{Xbal}$ and Notl and ligated together to obtain the pmirGL0-MITF-wt construct. The miR508-3p binding site in $3^{\prime}$ UTR of MITF region was mutated using a site-directed gene mutagenesis kit (Beyotime, Beijing, China) according to the manufacturer's instructions to obtain the pmirGL0-MITF-mut construct. All constructs were confirmed by sequencing.

\section{Cell culture and transfection}

Melanocytes were transfected with the pcDNA6.2-miR508-3p plasmid or the NC plasmid using lipofectamine 2000 (Invitrogen) according to the manufacturer's instructions. After 3 days of transfection, melanocytes were collected. Cell lysates and total RNA were prepared and subjected to western blot and quantitative real-time PCR analysis, respectively.

\section{Dual luciferase assay for microRNA target validation}

For the luciferase reporter assay, $2 \mu \mathrm{g}$ of pmirGL0-MITF-wt or pmirGLO-MITF-mut plasmid were co-transfected into 293T cells with miR508-3p plasmid or NC miRNA plasmid using lipofectamine 2000 (Invitrogen). Luciferase activities in

Table 1 Primers used in this study

\begin{tabular}{|c|c|c|}
\hline Primer name & Primer sequence $5^{\prime}$ to $3^{\prime}$ & Application \\
\hline MITF-F & TCCCAAGTCAAATGATCCAG & RT-PCR \\
\hline MITF-R & GAGCCTGCATTTCAAGTTCC & RT-PCR \\
\hline MITF-F2 & CGAGCTCCAGATGATCTACTTGCCTGAA & RT-PCR \\
\hline MITF-R2 & GCTCTAGATATCTGAACAGATTCACCGTCTACT & RT-PCR \\
\hline MITF-wt-F & CTAGTCTAGACAGATGATCTACTTGCCTGAA & Luciferase reporter-wt \\
\hline MITF-wt-R & ATAAGAATGCGGCCGCCTCGAGTATCTGAACAGATTCACCGTCTACT & Luciferase reporter-wt \\
\hline MITF-mut-F & CGGGCATGAAGATACAGTTCCTCCTCAACGAC & Luciferase reporter-mut \\
\hline MITF-mut-R & GTCGT TGAGGAGGAACTGTATCTTCATGCCCG & Luciferase reporter-mut \\
\hline miR508-3p-RT & GTCGTATCCAGTGCAGGGTCCGAGGTATTCGCACTGGATACGACTCATCT & RT-PCR \\
\hline miR508-3p-F & CGAGATTGTCACTTTTTGG & RT-PCR \\
\hline Common-R & CGAGCAGTGCAGGGTCCGAGGT & RT-PCR \\
\hline U6-RT & GTCGTATCCAGTGCAGGGTCCGAGGTATTCGCACTGGATACGACTCATCT & RT-PCR \\
\hline U6-F & CTCGCTTCGGCAGCACA & RT-PCR \\
\hline TYR-F & GCTTTAGCAACTTCATGGGA & RT-PCR \\
\hline TYR-R & CTTGTTCTTCTCTGGGACAC & RT-PCR \\
\hline TYRP2-F & GCCTTCTTTCTCCCTTC & RT-PCR \\
\hline TYRP2-R & CAGACCACTCGCCATT & RT-PCR \\
\hline$\beta$-actin- $\mathrm{F}$ & CTAAGGAGAAGGGCCAGTCC & RT-PCR \\
\hline$\beta$-actin-R & CTCAAGTTGGGGGACAAAAA & RT-PCR \\
\hline
\end{tabular}

$M I T F=$ microphthalmia transcription factor; $F=$ forward; $R=$ reverse; $R T=$ real time; $R T-P C R=$ reverse transcript $P C R ; w t=$ wild type; mut $=$ mutant; $T Y R=$ tyrosinase; TYRP2 = tyrosinase-related protein 2. 
the transfected cells were measured with a dual luciferase reporter assay kit (Promega, Fitchburg, WI, USA) 2 days after co-transfection. Firefly luciferase activity was normalized to renilla luciferase activity to adjust for transfection efficiency. Data are expressed as relative luciferase activities $(n=3$, mean $\pm \mathrm{SE})$.

\section{Quantitative real-time PCR for microRNA and messenger RNA}

Total RNA from melanocytes was extracted using TRIzol reagent (Invitrogen) or an RNeasy kit (Qiagen, Dusseldorf, Germany) according to the manufacturer's instructions and treated with DNase I (Sigma). For mRNA quantification, $1 \mu \mathrm{g}$ of total RNA was converted to CDNA using a CDNA synthesis kit (Takara, Dalian, China) according to the manufacturer's instructions, and quantitative real-time PCR was performed using SYBR Green PCR master mix (Takara). For miRNA quantification, CDNA was generated using a cDNA synthesis kit (Takara), a specific stem-loop RT primer and a common reverse primer according to a previously established method for real-time quantification of miRNAs (Chen et al., 2005), and quantitative real-time PCR was performed using SYBR Green PCR master mix. The primer sequences are listed in Table 1. Real-time PCR was performed on a 7500 Fast RealTime PCR ${ }^{\mathrm{TM}}$ system (Applied Biosystems, Foster City, CA). Melting curves for each sample were analyzed to validate specificity of amplification. All samples were run in triplicate, and the relative amount of miRNA and mRNA was normalized to the amount of $U 6$ and $\beta$-actin mRNA, respectively. Quantification of miRNA and mRNA transcript abundance was performed using the comparative threshold cycle $\left(C_{T}\right)$ method (Livak and Schmittgen, 2001).

\section{Western blot analysis}

Western blot analysis was performed as previously described (Dong et al., 2012) with the following primary antibodies: rabbit anti-MITF at $1: 200$ dilution (Santa Cruz, Finnell Stree tDallas, Texas, USA), rabbit anti-TYR at 1:1000 dilution (Santa Cruz), rabbit anti-TYRP1 at 1:1000 dilution (Abcam, Cambridge, UK), rabbit anti- $\beta$-actin (Sigma). Anti-rabbit lgG secondary antibody was purchased from Invitrogen. Immunoblots were scanned on a ChemiDOC ${ }^{\mathrm{TM}} \mathrm{XRS}^{+}$imager (BioRad, Hercules, CA), and protein levels were quantified using the Image-Pro Plus software (Olympus, Tokyo, Japan).

\section{Immunocytochemistry}

Melanocytes were washed three times in $0.1 \mathrm{M}$ PBS for $3 \mathrm{~min}$ each, fixed in $4 \%$ paraformaldehyde, and then incubated at room temperature in $3 \%$ hydrogen peroxide for $15 \mathrm{~min}$ to block the action of any endogenous peroxidase. After washing with $0.1 \mathrm{M}$ PBS three times for $5 \mathrm{~min}$ each, cells were immersed in BSA (Bovine Serum Albumin) at $37^{\circ} \mathrm{C}$ for $40 \mathrm{~min}$. Cells were then incubated at $4^{\circ} \mathrm{C}$ overnight in antiMITF antibody solution. Following washing three times in $0.1 \mathrm{M}$ PBS for $5 \mathrm{~min}$ each, cells were incubated with HRP (Horseradish Peroxidase)-conjugated anti-rabbit lgG for $30 \mathrm{~min}$ at $37^{\circ} \mathrm{C}$, and then incubated with the substrate
$3,3^{\prime}-D A B$ for $20 \mathrm{~min}$ at room temperature to produce a brown precipitate. The immunostaining of the cells was then observed under a microscope (Leica, Germany). For NCs, PBS was used to substitute the primary antibody.

\section{Melanin measurement}

Melanocytes were harvested and rinsed with PBS followed by addition of I $\mathrm{ml}$ of $1 \mathrm{~N} \mathrm{NaOH}$ to dissolve the melanin. For assay of alkali-soluble melanin, melanin content was measured spectrophotometrically at an absorbance of $475 \mathrm{~nm}$. For the spectrophotometric assay of eumelanin, cells were hydrolyzed in hot 30\% hypophosphoric acid and hydroiodic acid. After cooling, 50\% ethanol was added and cell samples were centrifuged at $2234 \times \mathbf{g}$ for $10 \mathrm{~min}$. Insoluble eumelanic pigments were selectively solubilized in hot sodium hydroxide and hydrogen peroxide; they were cleared by centrifugation at $10700 \times \mathbf{g}$ for 1 min with a Sorvall Ultracentrifuge (Waltham, Massachusetts, USA). Supernatants were analyzed for absorbance at $350 \mathrm{~nm}$. For the spectrophotometric assay of pheomelanins, cells were solubilized in a phosphate buffer (pH 10.5) and cleared by centrifugation at $10700 \times \mathbf{g}$ for $10 \mathrm{~min}$. and then analyzed for absorbance at $400 \mathrm{~nm}$. The melanin measurement was normalized to total amount of cells. All experiments were performed in triplicate.

\section{Statistical analysis}

Data, which were reported as mean \pm SE from three replicates, were analyzed using ANOVA and Fisher's protected LSD test. The differences in abundance of miRNA, mRNA, protein, relative luciferase activities and melanin production between control and experimental groups were determined by ANOVA using SPSS 11.5 software (SPSS, Chicago, IL, USA).

\section{Results}

miR508-3p targets the predicted microRNA binding site in the $3^{\prime}$ UTR of microphthalmia transcription factor

To validate the specificity of miR508-3p targeting MITF through the predicted miRNA binding site in $3^{\prime}$ UTR of MITF with high similarity among camel, horse, cattle and human (Figure $1 \mathrm{a}$ and $\mathrm{b}$ ), luciferase reporter assays were performed using luciferase reporter constructs containing either the wild type MITF (pmirGL0-sGC-wt) or the mutant MITF (pmirGLOsGC-mut). The constructs were co-transfected into 293T cell with the miR508-3p expression plasmid or NC plasmid. Dual luciferase reporter assays showed that the reporter activity in cells co-transfected with pmirGL0-MITF-wt and miR508-3p plasmid was decreased by $>40 \%$, compared with the cells co-transfected with pmirGLO-MITF-wt and the NC plasmid (Figure 1c). Luciferase reporter activity in cells co-transfected with pmirGL0-MITF-mut and miR508-3p plasmid was similar to the reporter activity in cells co-transfected with pmirGLOMITF-mut and the NC miRNA plasmid (Figure 1d). These data indicate that miR508-3p can bind and regulate MITF in a sequence specific fashion through the predicted binding site in $3^{\prime}$ UTR of MITF. 
(a)

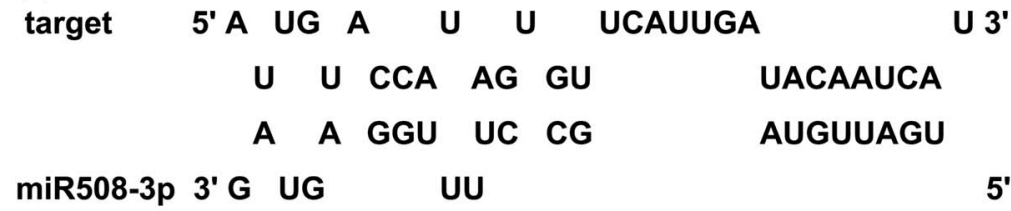

(b)

$\begin{array}{ll}\text { Camel } & \text { ACCAUAGUGUUCAUUGAUACAAUCAUACCAUUG } \\ \text { Human } & \text { ACCAUAGUGUUCAUUGAUACAAUCAUAGCAUUG } \\ \text { Bos taurus } & \text { ACCAUAGUGUUCAUUGAUACAAUCAUAGCAUUG } \\ \text { Chimpanzee } & \text { ACCAUAGUGUUCAUUGAUACAAUCAUAGCAUUG } \\ \text { Horse } & \text { ACCAUAGUGUUCAUUGAUACAAUCAUACCAUUG }\end{array}$

(c)

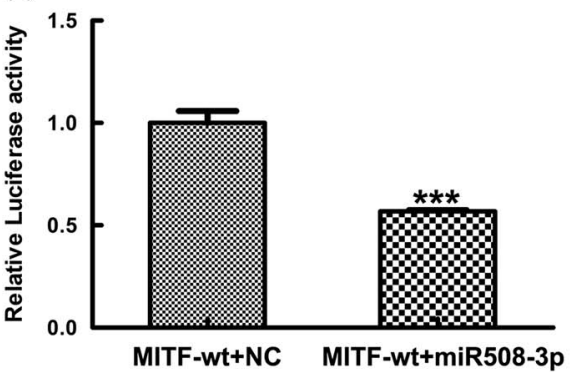

(d)

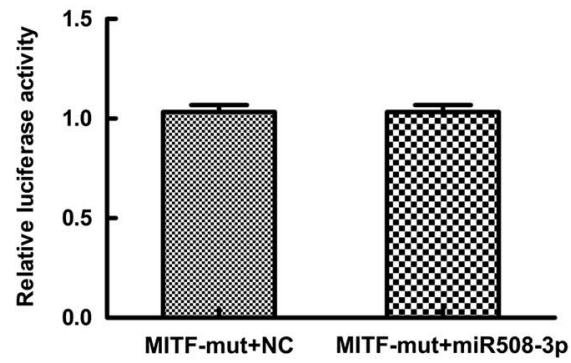

Figure 1 Specificity of miR508-3p targeting 3'UTR of microphthalmia transcription factor (MITF) transcript. (a) Predicted miR508-3p binding site in the 3'UTR of MITF transcript. (b) Alignment of MITF sequences around the miR508-3p binding site (the highlighted boxes indicate the miR508-3p seed region). (c) Luciferase reporter activities in 293T cells co-transfected with the reporter construct containing the wild type (wt) 3'UTR of MITF (pmirGL0-MITF-wt) and the miR508-3p or the negative control (NC) plasmid. (d) Luciferase reporter activities in 293T cells co-transfected with the mutant reporter construct (pmirGL0-MITF-mut) and the miR508-3p or the NC plasmid.
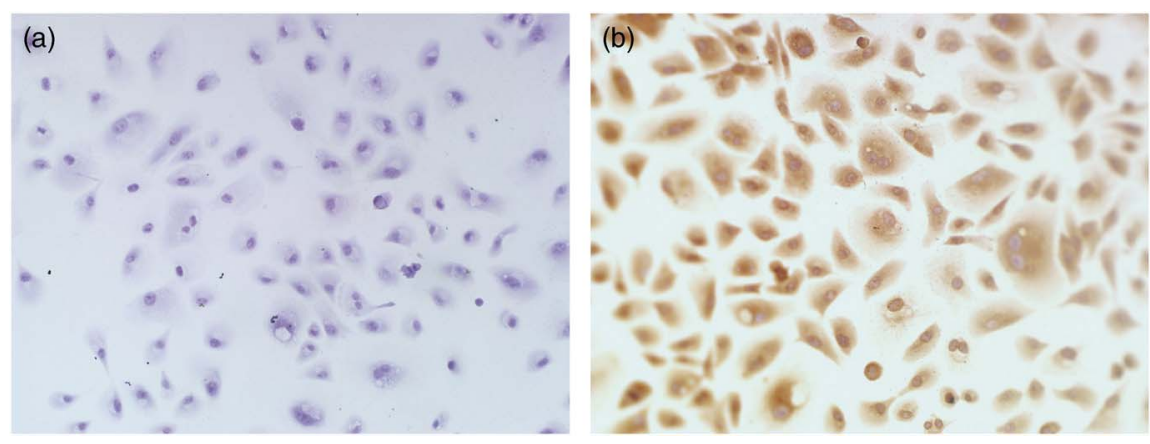

Figure 2 Localization of miR508-3p in alpaca melanocytes by in situ hybridization analysis. (a) Melanocytes hybridized with negative control probe. (b) Melanocytes hybridized with digoxigenin-labeled miR508-3p probe.

Localization of miR508-3p in alpaca melanocytes

To identify the localization of miR508-3p in alpaca melanocytes in vitro, in situ hybridization was performed. The assay showed that no specific hybridization signal was detected in the cells hybridized with the NC probe (Figure 2a) and specific hybridization signal for miR508-3p in the cytoplasm of alpaca melanocytes (Figure 2b).

Effect of miR508-3p overexpression on messenger RNA and protein abundance of microphthalmia transcription factor To determine whether MITF is a target of miR508-3p, the miR508-3p expression plasmid was used to transfect alpaca melanocytes to test the effect of miR508-3p on expression of
MITF. Quantitative real-time PCR analysis showed increased accumulation of miR508-3p in melanocytes transfected with the miRNA expression plasmid in comparison with the cells transfected with the NC plasmid ( $P<0.01$, Figure 3a). Overexpression of miR508-3p resulted in a decrease in the abundance of both MITF mRNA $(P<0.01$, Figure $3 \mathrm{~b})$ and protein as analyzed by western blotting and immunocytohistology (Figure $3 \mathrm{C}$ to e). These data indicate that MITF is regulated by miR508-3p.

Effect of miR508-3p overexpression on the expression of melanogenic genes

The abundance of both mRNA and protein for TYR and TYRP2 genes was examined in melanocytes transfected with 
(a)

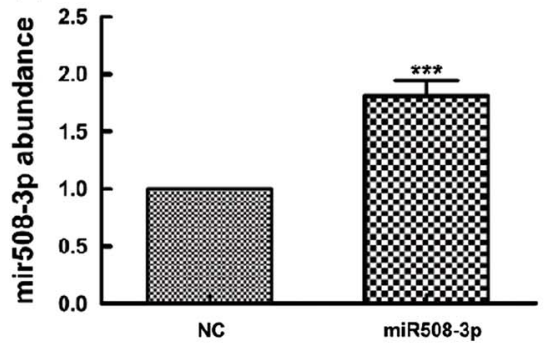

(c)

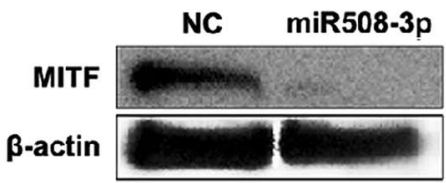

(b)

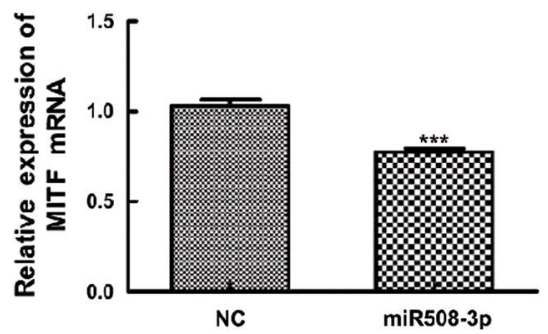

(d)

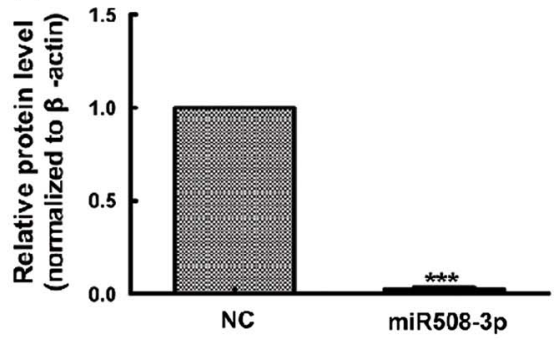

(e)
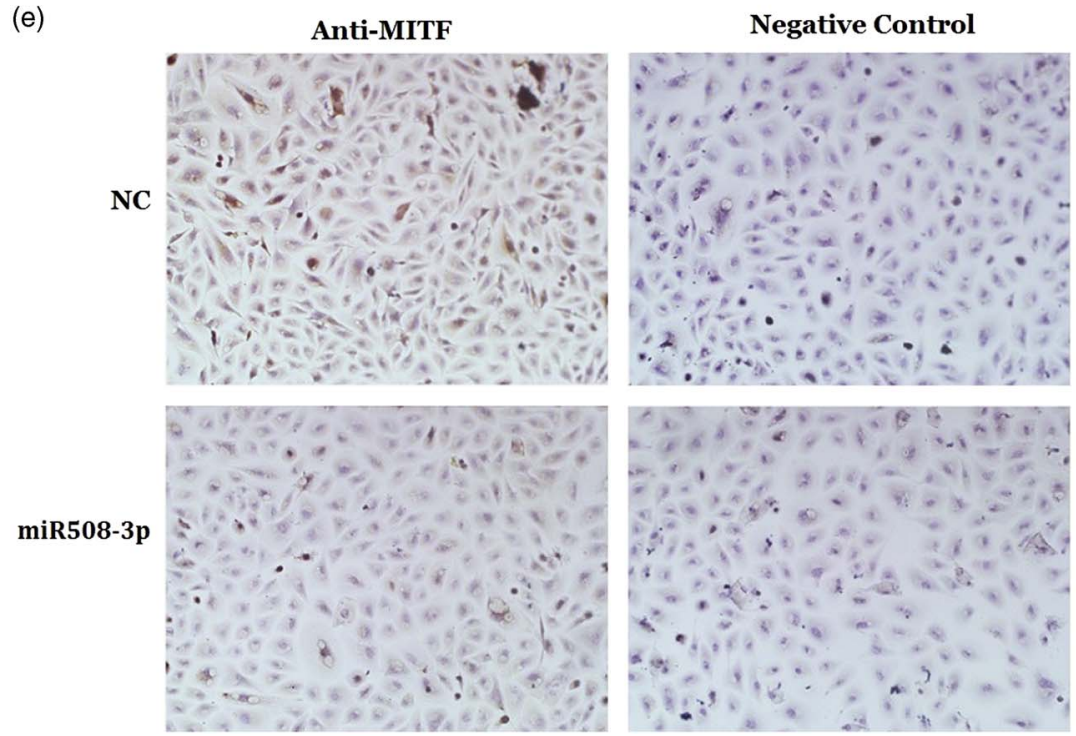

Figure 3 Effect of miR508-3p on messenger RNA (mRNA) and protein abundance of microphthalmia transcription factor (MITF). (a) miR508-3p expression in melanocytes transfected with the miR508-3p expression plasmid. (b) MITF mRNA expression in melanocytes transfected with the miR508-3p expression plasmid. (c-e) MITF protein expression in melanocytes transfected with the miR508-3p expression plasmid analyzed using Western blot and immunocytochemical detection, respectively. Data are expressed as mean $\pm \mathrm{SE}$ from three replicates. ${ }^{* * *} P<0.001$. NC $=$ negative control.

the miR508-3p expression construct relative to cells transfected with the NC plasmid. As shown in Figure 4a, overexpression of miR508-3p in melanocytes significantly decreased the mRNA abundance for TYR and TYRP2 genes $(P<0.01$ and $P<0.05$, respectively). Western blot analysis showed that the expression of TYR and TYRP2 proteins was significantly reduced in melanocytes overexpressing miR508-3p $(P<0.01$, Figure $4 b$ and $c)$.

\section{Effect of miR508-3p overexpression on the melanin production}

To determine whether miR508-3p overexpression affects melanin production, the production of total alkali-soluble melanogenesis (ASM), eumelanogenesis (EM) and pheomelanogenesis (PM) were measured in alpaca melanocytes transfected with the miR508-3p expression plasmid. As shown in Figure 5, overexpression of miR508-3p in alpaca melanocytes reduced ASM production by $45 \%(P<0.01$, Figure $5 a)$, EM production by about $45 \%(P<0.01$, Figure $5 b)$ and PM production by about $60 \%(P<0.01$, Figure $5 c)$.

\section{Discussion}

Melanocytes are specialized cells in which melanin synthesis (eumelanins and pheomelanins) takes place. Melanin is responsible for skin and hair color in mammalian species. The phenotype of coat color depends on two types of melanin (black to brown eumelanin and yellow to reddish brown 
(a)

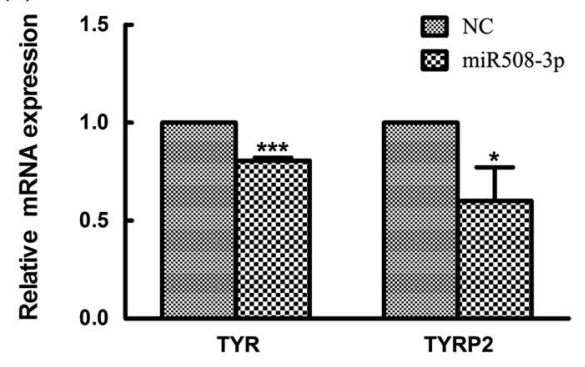

(c)

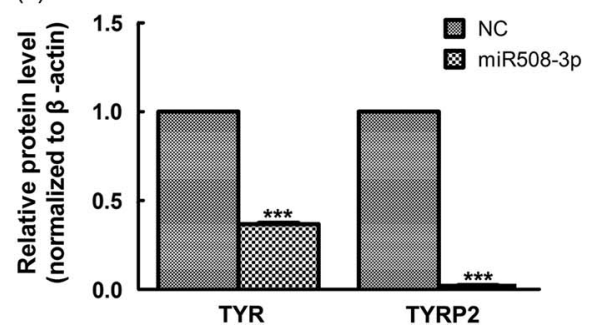

(b)

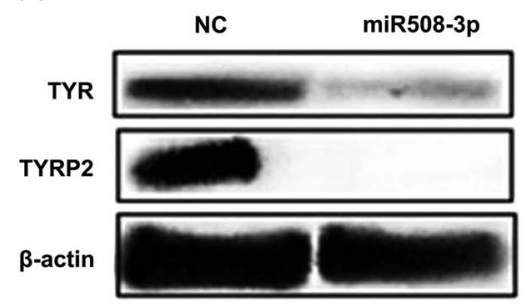

Figure 4 Effect of miR508-3p on the expression of coat color genes in melanocytes. (a) tyrosinase (TYR) and tyrosinase-related protein 2 (TYRP2) messenger RNA (mRNA) expression in melanocytes transfected with the miR508-3p expression plasmid. (b and c) TYR and TYRP2 protein expression in melanocytes transfected with the miR508-3P expression plasmid. Data were normalized to $\beta$-actin and expressed as relative fold change. Data are expressed as mean \pm SE from three replicates. ${ }^{*} P<0.05 ;{ }^{* * *} P<0.001$. NC $=$ negative control.

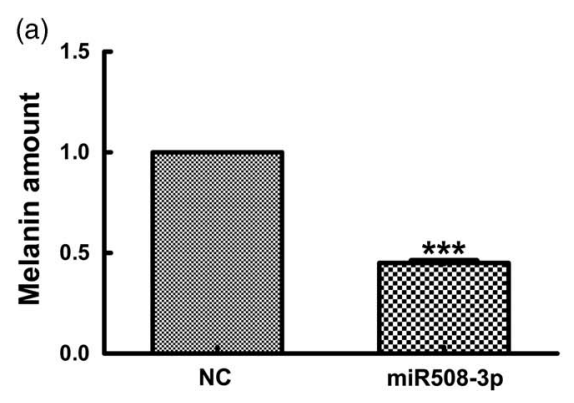

(c)

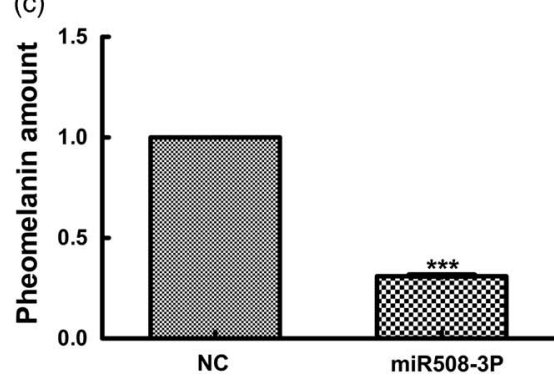

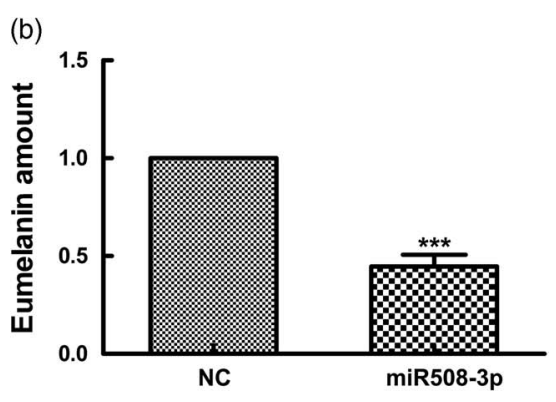

Figure 5 Effect of miR508-3p on melanin production. (a) Alkali total melanin production in melanocytes overexpressing miR508-3p. (b) Eumelanin production in melanocytes overexpressing miR508-3p. (c) Pheomelanin production in melanocytes overexpressing miR508-3p. Data are expressed as mean \pm SE from three replicates. ${ }^{* \star} P<0.001$. NC $=$ negative control.

pheomelanin) produced by melanocytes resident in the skin (Ito et al., 2000; Ito and Wakamatus, 2008). The quality and ratio of eumelanin to pheomelanin dictate the coat color (Ito et al., 2000). Despite considerable knowledge of the genetic regulation of coat color in mice and identification of loci involved in coat color regulation in fiber-producing species, the molecular and cellular mechanisms that regulate coat color are not completely understood. A number of miRNAs involved in melanogenesis have been reported in different animal species. Such miRNAs include miR-137 in mouse (Dong et al., 2012), miR-429 in fish (Yan et al., 2013) and miR-8 in Drosophila (Kennell et al., 2012). In order to identify target genes of miRNA, which are specifically associated with hair color formation, we have screened miRNA expression profile in alpaca skin. As a result, several miRNA that are differentially expressed in skin were identified, such as miR508-3p (Tian et al., 2012). In addition, the functional role of miRNA on melanogenesis was also investigated, highlighted with the 
studies on the effect of Ipa-miR-nov-66 (Yang et al., 2015) and miR-25 (Zhu et al., 2010) on melanin production.

Melanogenesis is mainly determined by genes on several pathways such as the traditional c-kit and cyclic AMP (cAMP) pathways, which merge at MITF (reviewed by Tachibana, 2000). MITF is directly regulated by miRNAs such as miR-137 (Dong et al., 2012) and miR-25 (Zhao et al., 2015). MITF is one of the target genes for miR508-3p. In general, the actions of miRNA are to promote degradation of mRNA or to suppress mRNA translation by binding to the $3^{\prime}$ UTR of target mRNA (Bartel, 2004; Wu et al., 2006). Results of the luciferase reporter assay in the present study provided evidence indicating that MITF is a target gene regulated by miR508-3p.

miR508-3p is expressed higher in alpaca skins with white hair color than that with black hair color (Tian et al., 2012). Here, we first found that miR508-3p was expressed in cytoplasm of alpaca melanocytes with high abundance, which suggested that it may play a role in melanocyte. Overexpression of miR508-3p in alpaca melanocytes down-regulated the expression of MITF both at the transcriptional and translational level, especially at translational level. Moreover, we found the transcriptional and transcriptional expression of TYR and TYRP2 were decreased by miR508-3p targeting MITF. The melanogenic enzymes TYR, TYRP1 and TYRP2 are regulated by the transcription factor MITF for the production of melanin (Widlund and Fisher, 2003; Levy et al., 2006). However, it has been reported that there is no transcriptional regulation of TYR, TYRP1 and TYRP2 by the CAMP pathway in human melanocytes (Busca and Ballotti, 2000). So, the results suggests that the regulation of miR508-3p on the melanogenesis by targeting MITF may not be through the CAMP pathway.

It was reported that MITF is not a principal regulator of the TYRP2 gene (Tachibana, 2000). TYR is absolutely required for both eumelanin and pheomelanin synthesis, whereas TYRP1 and TYRP2 seem to be more crucial for eumelanin synthesis (Busca and Ballotti, 2000). Here, we found that eumelanin production was decreased $45 \%$ by miR508-3p overexpression. Therefore, decreased eumelanin synthesis in cells overexpressing miR508-3p is due to lower expression of TYR and TYRP2, and decreased pheomelanin synthesis is due to reduced expression of TYR. This finding is similar to the observation that agouti signal protein decreases eumelanin synthesis but promotes pheomelanin synthesis in melanocytes, due to inhibition of TYR activity and the loss of TYRP1 and TYRP2 expression (Kobayashi et al., 1995).

In conclusion, we provided evidence supporting an important functional role for miR508-3p in alpaca melanocytes by targeting 3'UTR of MITF. The post-transcriptional expression of TYR and TYRP2 is decreased consequently, thereby resulting in decreased melanin production. The observed effect of miR508$3 p$ on melanin production is via down-regulation of MITF expression, suggesting a functional role for miR508-3p in the regulation of melanogenesis. Hence, miR508-3p is a potential candidate gene for producing natural coat colors in fiberproducing species by transgenic approach.

\section{Acknowledgments}

This work was supported by a grant from the National Natural Science Foundation of China (NSFC, No. 31201868), 131 expert project of Shanxi Province, Special Fund for Agro-Scientific Research in the Public Interest of China (No. 201303119) and Youth Foundation of Shanxi Province (2014021028-2).

\section{References}

Andl T, Murchison EP, Liu F, Zhang Y, Yunta-Gonzalez M, Tobias JW, Andi CD, Seykora JT, Hannon GT and Millar SE 2006. The miRNA-processing enzyme dicer is essential for the morphogenesis and maintenance of hair follicles. Current Biology 16, 1041-1049.

Bai R, Sen A, Yu Z, Yang G, Wang H, Fan R, Lv L, Lee KB, Smith GW and Dong C 2010. Validation of methods for isolation and culture of alpaca melanocytes: a novel tool for in vitro studies of mechanisms controlling coat color. Asian Australia Journal Animal Science 23, 430-436.

Bartel DP 2004. MicroRNAs: genomics, biogenesis, mechanism, and function. Cell 116, 281-297.

Bemis LT, Chen R, Amato CM, Classen EH, Robinson SE, Coffey DG, Erickson PF, Shellman YG and Robinson WA 2008. MicroRNA-137 targets microphthalmiaassociated transcription factor in melanoma cell lines. Cancer Research 68, 1362-1368.

Busca R and Ballotti R 2000. Cyclic AMP a key messenger in the regulation of skin pigmentation. Pigment Cell Research 13, 60-69.

Chen C, Ridzon DA, Broomer AJ, Zhou Z, Lee DH, Nguyen JT, Barbisin M, Xu NL, Mahuvakar VR, Andersen MR, Lao KQ, Livak KJ and Guegler KJ 2005. Real-time quantification of microRNAs by stem-loop RT-PCR. Nucleic Acids Research 33, e179.

Dong C, Wang H, Xue L, Dong Y, Yang L, Fan R, Yu X, Tian X, Ma S and Smith GW 2012. Coat color determination by miR-137 mediated down-regulation of microphthalmia-associated transcription factor in a mouse model. RNA 18, 1679-1686.

Goswami S, Tarapore RS, TeSlaa JJ, Grinblat Y, Setaluri V and Spiegelman VS 2010. MicroRNA-340-mediated degradation of microphthalmia-associated transcription factor mRNA is inhibited by the coding region determinantbinding protein. Journal of Biological Chemistry 285, 20532-20540.

Ito $S$ and Wakamatus K 2008. Chemistry of mixed melanogenesis-pivotal roles of dopaquinone. Photochemistry and Photobiology 84, 582-592.

Ito T, Tashiro K, Muta S, Ozawa R, Chiba T, Nishizawa M, Yamamoto K, Kuhara S and Sabaki $Y$ 2000. Toward a protein-protein interaction map of the budding yeast: a comprehensive system to examine two-hybrid interactions in all possible combinations between the yeast proteins. Proceedings of the National Academy of Sciences of the United States of America 97, 1143-1147.

Kennell JA, Cadigan KM, Shakhmantsir I and Waldron EJ 2012. The microRNA miR-8 is a positive regulator of pigmentation and eclosion in Drosophila. Developmental Dynamics 241, 161-168.

Kobayashi T, Viera WD, Potterf B, Sakai C and Imokawa G 1995. Modulation of melanogenic protein expression during the switch from eu- to pheomelnogenesis. Journal of Cell Science 108, 2301-2309.

Levy C, Khaled M and Fisher DE 2006. MITF: master regulator of melanocyte development and melanoma oncogene. Trends in Molecular Medicine 12, 406-414. Livak KJ and Schmittgen TD 2001. Analysis of relative gene expression data using real-time quantitative PCR and the $2^{-\Delta \Delta C T}$ method. Methods 25, 402-408.

Segura MF, Hanniford D, Menendez S, Reavie L, Zou X, Alvarez-Diaz S, Zakrzewski J, Blochin E, Rose A, Bogunovic D, Polsky D, Wei J, Belitskaya-Levy J, Lee P, Bhardwaj N, Osman I and Hernando E 2009. Aberrant miR-182 expression promotes melanoma metastasis by repressing FOXO3 and microphthalmiaassociated transcription factor. Proceedings of the National Academy of Sciences 106, 1814-1819.

Tachibana M 2000. MITF: a stream flowing for pigment cells. Pigment Cell Research 13, 230-240.

Tian X, Jiang J, Fan R, Wang H, Meng X, He X, He J, Geng J, Yu X, Song Y, Zhang D, Yao J, Smith GW and Dong C 2012. Identification and characterization of microRNAs in white and brown alpaca skin. BMC Genomics 13, 555-566. 


\section{Functional role of miR508-3p in melanogenesis}

Vachtenheim J and Borovanský J 2010. 'Transcription physiology' of pigment formation in melanocytes: central role of MITF. Experimental Dermatology 19, 617-627.

Wenguang Z, Jianghong W, Jinquan L and Yashizawa M 2007. A subset of skin-expressed microRNAs with possible roles in goat and sheep hair growth based on expression profiling of mammalian microRNAs. Omics: A Journal of Integrative Biology 11, 385-396.

Widlund HR and Fisher DE 2003. Microphthalamia-associated transcription factor: a critical regulator of pigment cell development and survival. Oncogene 22, 3035-3041.

Wu L, Fan J and Belasco JG 2006. MicroRNAs direct rapid deadenylation of mRNA. Proceedings of the National Academy of Sciences of the United States of America 103, 4034-4039.

Yan B, Liu B, Zhu CD, Li KL, Yue ப, Zhao JL, Gong XL and Wang CH 2013. microRNA regulation of skin pigmentation in fish. Journal of Cell Science 126, 3401-3408.

Yang S, Fan R, Shi Z, Ji K, Zhang J, Wang H, Herrid M, Zhang Q, Yao J, Smith GW and Dong C 2015. Identification of a novel microRNA important for melanogenesis in alpaca (Vicugna pacos). Journal of Animal Science 93, 1622-1631.
Yi R, O'Carroll D, Pasolli HA, Zhang Z, Dietrich FS, Tarakhovsky A and Fuchs E 2006. Morphogenesis in skin is governed by discrete sets of differentially expressed microRNAs. Nature Genetics 38, 356-362.

Zhai Q, Zhou L, Zhao C, Wan J, Yu Z, Guo X, Qin J, Chen J and Lu R 2012. Identification of miR508-3p and miR-509-3p that are associated with cell invasion and migration and involved in the apoptosis of renal cell carcinoma. Biochemical and Biophysical Research Communications 419, 621-626.

Zhao Y, Wang P, Meng J, Ji Y, Xu D, Chen T, Fan R, Yu X, Yao J and Dong C 2015. MicroRNA-27a-3p inhibits melanogenesis in mouse skin melanocytes by targeting Wnt3a. International Journal of Molecular Sciences 16, 10921-10933.

Zhou L, Chen J, Li Z, Li X, Hu X, Huang Y, Zhao X, Liang C, Wang Y, Sun L and Shi M 2010. Integrated profiling of microRNAs and mRNAs: microRNAs located on Xq27. 3 associate with clear cell renal cell carcinoma. PloS One 5, e15224.

Zhu Z, He J, Jia X, Jiang J, Bai R, Yu X, Lv L, Fan R, He X, Geng J, Dong Y, Qiao D, Lee KB, Smith GW and Dong C 2010. MicroRNA-25 functions in regulation of pigmentation by targeting the transcription factor MITF in alpaca (Lama pacos) skin melanocytes. Domestic Animal Endocrinology 38, 200-209. 